\title{
The Grasshopper Median Neuroblast Is a Multipotent Progenitor Cell That Generates Glia and Neurons in Distinct Temporal Phases
}

\author{
Barry G. Condron and Kai Zinn \\ Division of Biology, California Institute of Technology, Pasadena, California 91125
}

The midline of the insect CNS provides a good system for studying the control of neuronal and glial cell fates, because it contains a small number of distinct cell types that arise from a unique set of precursors. In this report we analyze the development of the CNS midline in the grasshopper embryo, focusing on the median neuroblast (MNB) cluster, which contains the majority of midline neurons. We have directly traced cell lineage in the MNB cluster by injecting the MNB with tracer dyes and following the development of its progeny in whole embryo cultures. As least three types of neuronal progeny are labeled with tracer dye in these experiments. In addition, we find that all of the midline glia are also derived from the MNB. Thus, this neuroblast is actually a multipotent progenitor cell. We also examined the temporal and spatial patterns of midline development by staining embryos with antibodies to neuronal and glial markers and to the protein product of the engrailedgene, which is transiently expressed by all MNB progeny. Our data show that neuronal and glial progeny are generated from the MNB in distinct temporal phases. A change in the orientation of the MNB's mitotic spindle correlates with the transition between two of the phases of progeny production.

[Key words: insect nervous system development, CNS midline, lineage tracing, glia, engrailed]

The development of the CNS midline has been intensively studied in both orthopterans (the grasshopper Schistocerca and the locust Locusta migratoria) and dipterans (the fruit fly Drosophila melanogaster). The CNS midline in all of these embryos lies between the paired longitudinal tracts. It is derived from the mesectoderm, which is located at the boundary between mesoderm and lateral neuroepithelium in the blastoderm embryo. Mesectodermal cells have morphologies and patterns of gene expression that are different from those of lateral neuroepithelial cells, and a distinct group of "midline genes" controls the development of the CNS midline (Klambt et al., 1991). Thus, the insect CNS midline provides a good system in which to study

\footnotetext{
Received Jan. 13, 1994; revised Mar. 23, 1994; accepted Mar. 28, 1994.

We thank Michael Bastiani for invaluable help with the embryo culture system and with injection methods, Scott Fraser for the use of injection equipment, and Gilles Laurent for the use of his grasshopper colony. We also thank Scott Fraser, Ioshua Sanes, Nipam Patel, David Anderson, Corey Goodman, Sarah Fashena, and S. Stans for helpful discussions. This work was supported by National Institutes of Health Grant NS28182 to K.Z., as well as by a Pew Scholars Award, a McKnight Scholars Award, and the Markey Foundation. B.G.C. was supported by a Del Webb postdoctoral fellowship.

Copyright (c) 1994 Society for Neuroscience $0270-6474 / 94 / 145766-12 \$ 05.00 / 0$
}

the cellular and molecular mechanisms involved in cell fate determination and axon guidance in the nervous system.

Orthopteran embryos, in which the midline cells are large and can be clearly visualized, have a pattern of midline neurons similar to that found in the genetically tractable but much smaller Drosophila embryo. In each segment of the orthopteran CNS, one of the mesectodermal cells becomes the median neuroblast (MNB), which undergoes reductive division to generate a chain of ganglion mother cells (GMCs). Other mesectodermal cells become midline precursors (MPs). MPs and GMCs divide only once to generate two neuronal progeny. The vast majority of midline neurons are derived from the MNB. By the end of embryogenesis, the MNB cluster in the metathoracic ganglion contains about $80-90$ neuronal progeny. About 20 of these are efferent dorsal unpaired median (DUM) neurons, which are octopaminergic neuromodulatory motorneurons. The remainder of the neurons in the MNB cluster are intersegmental and local interneurons (Thompson and Siegler, 1991).

In addition to these neurons, the CNS midline also produces several types of glia. These midline glia have not been well characterized in orthopteran embryos, but it has been assumed that they are derived from a distinct set of glial progenitor cells. In Drosophila, three pairs of axonal midline glia that may be essential for the correct formation of the commissural tracts have been described (Jacobs and Goodman, 1989; Klambt et al., 1991).

In order to understand the processes involved in development of the CNS midline, it is important to determine the lineages of midline cells. We have studied cell lineage in the MNB cluster by filling the MNB with tracer dyes and examining the pattern of progeny produced in whole embryo cultures. This method has been used successfully in many other systems to study cell lineages, but it has not previously been applied to orthopteran embryos. As expected, we find that several classes of midline neurons are generated from the MNB. Surprisingly, however, midline glia are labeled with tracer dyes in these experiments, indicating that they are also derived from the MNB. The neurons and glia are generated from the MNB in distinct temporal phases, and the transition between the glial and second neuronal phases of progeny production is correlated with a change in the direction of MNB cytokinesis. We suggest that this change in cytokinesis may be involved in the determination of cell fate within the lineage.

\section{Materials and Methods}

Embryo cultures and dye injections. Grasshopper embryos were staged according to Bentley et al. (1979). Embryo cultures were set up and maintained essentially as described by Myers and Bastiani (1993b). Under these conditions, embryos develop in a normal manner from 
$32-35 \%$ to over $45 \%$ in about $72 \mathrm{hr}$ at $33^{\circ} \mathrm{C}$. Cells are injected with a mixture of lysinated rhodamated dextran $10 \mathrm{k}$ and lysinated biotinylated dextran 10k (Molecular Probes, D1817 and D1956, respectively) at concentrations of $20 \mathrm{mg} / \mathrm{ml}$ in water. The rhodamine dextran can be replaced with fluorescein dextran. Lot-to-lot variability of the toxicity of these tracers is removed by spin filtration with an Amicon 10k cutoff filter. The retentate is used. Other tracer dyes were extensively tested before the two described were chosen. These had the following problems: HRP, difficult to inject; phycocyanins, unstable; 3K dextran tracers, poor fixability; dual-labeled biotin-rhodamine dextrans, poor fixability. From $20 \%$ to $50 \%$ of injected cells develop correctly in embryos that display normal overall development. The MNB can be successfully injected only in the period between $28 \%$ and $35 \%$ of development (because of the anterior-to-posterior developmental gradient, an A4 MNB in a $32 \%$ embryo is at about $28 \%$ of development). Before $28 \%$, the viability of injected cells is poor. After $35 \%$, the MNB is surrounded by a glial sheath. Injection through this sheath usually kills the glia, and this apparently leads to death or abnormal development of the injected MNB.

Histochemistry. Embryos were fixed as described by Patel et al. (1989). The following antibodies were used for staining: (1) 8B7: this MAb was isolated and characterized by Dr. Michael Bastiani, and was provided by him as ascites fluid, which was used at a dilution of $1: 300 ;(2)$ inv4D9: this was provided by Dr. Nipam Patel (Patel et al., 1989) and was used at a dilution of $1: 60 ;$ (3) $7 \mathrm{H} 7$ was provided by Dr. N. Patel (Bastiani et al., 1992; Singer et al., 1992) and was used at a dilution of 1:60; (4) the $\beta$-tubulin antibody was from Amersham and was used at a dilution of $1: 300 ;(5)$ anti- $\beta$-galactosidase antibodies were from Promega and were used at 1:300. The secondary antibody was goat anti-mouse, HRP or FITC conjugated, and was obtained from Jackson ImmunoResearch (111-035-003 and 111-095-003, respectively). For detection of the biotin dextran tracer, streptavidin-HRP was used (Molecular Probes, S-911); $0.25 \mu \mathrm{g}$ were incubated in $200 \mathrm{ml}$ of PBT with dissected CNS preparations for $2 \mathrm{hr}$ at room temperature. HRP histochemistry with $\mathrm{DAB}$ (to generate brown staining), or $\mathrm{DAB}+\mathrm{Ni}$ (to generate black staining) was performed as described (Patel et al., 1989). All samples were mounted in glycerol and viewed through a Zeiss Axioplan microscope or a Bio-Rad 600 confocal microscope.

\section{Results}

Tracing cell lineage at the CNS midline

We examined cell lineage relationships in the MNB cluster by filling the MNB with dye and analyzing the pattern of labeled progeny after culturing the whole embryo. We used lysinated rhodamine and biotin tracer dyes conjugated to a $10,000 \mathrm{M}_{r}$ dextran, which blocks dye movement through gap junctions and prevents its transfer to uninjected cells. The development of the lineage can be observed in living embryos by visualization of rhodamine fluorescence, and at the end of an experiment the embryos are fixed and the dye visualized using fluorescence or HRP histochemistry. This technique has been used successfully in many other systems to study cell lineages (Gimlich and Braun, 1985; Bronner-Fraser and Fraser, 1988; Copenhaver, 1993), but has not been previously applied to grasshopper embryo cultures. We normally injected MNBs in segments T3 to A4 of $32-35 \%$ grasshopper embryos. During the $72 \mathrm{hr}$ culture period, the embryos advance through about $10 \%$ of development. There is an anterior-to-posterior developmental gradient of about $1 \%$ per segment, so our technique allows examination of the MNB lineage between $28 \%$ and $45 \%$ of development. All axon pathways examined have been found to develop normally in cultured embryos (Myers and Bastiani, 1993a,b; B. G. Condron and K. Zinn, unpublished observations). The percentages cited below in descriptions of specific midline developmental events are all $\pm 1 \%$, because there is some variability in timing between embryos.

Shortly after injection, dye was confined to the injected MNB (Fig. $1 G$ ). When injected embryos were cultured for $72 \mathrm{hr}$ prior to fixation, a large number of labeled progeny cells were observed. The cell bodies of MNB progeny neurons are located just anterior to the MNB and are labeled with biotin (Fig. 1A). The biotin-labeled axons observed in Figure $1 A$ (arrow and arrowhead) extend anteriorly along the median fiber tract (MFT) and follow two different bifurcating pathways. One set bifurcates in the anterior commissure, indicating that these are likely to be axons of efferent DUM neurons (Goodman, 1982); in this study, we denote neurons whose axons follow this pathway as "MP4-class," because this pathway is pioneered by neurons that were thought to be MP4 progeny. The other set bifurcates in the posterior commissure and then follows a diagonal pathway, terminating in a club-shaped ending between the commissures. There are a number of other pathways that bifurcate in the posterior commissure that can also be observed in these experiments (see Fig. 2D). The first neurons whose axons bifurcate in the posterior commissure were thought to be MP6 progeny, and in this study we denote all neurons of this type as "MP6class." We find that dye-labeled MP4-class and MP6-class axons often appear simultaneously, suggesting that a single division of a GMC may sometimes generate one neuron of each type.

In addition to neurons, we also observed dye-labeled cell bodies anterior to the MNB cluster in cultured embryos. These labeled cells do not have axons, and are located in positions characteristic of midline glia (Fig. $1 A, B, H$ ). To determine whether they are glia, we staincd dyc-injected cultured embryos with $\mathrm{MAb} 7 \mathrm{H} 7$, which recognizes the plasma membrane protein annulin (Singer et al., 1992). After 40\%, annulin expression in the CNS is restricted to midline glia (Bastiani et al., 1992), and we have found that $7 \mathrm{H} 7$ stains most or all glial processes. It is thus a good marker for observation of the development and morphology of midline glia.

In Figure $1 B$, the pattern of rhodamine-dextran-labeled MNB progeny $(r e d)$ is visualized by confocal microscopy. Figure $1 C$ shows the pattern of annulin staining (green) of the same cells, as detected with fluorescein-conjugated second antibody. Superimposition of the two images (Fig. $1 D$ ) clearly shows that all of the annulin-staining glial cells are also labeled with dye (yellow regions), indicating that these glia are progeny of the MNB. Thus, the lineage tracing data show that the MNB is pluripotential and gives rise to both neurons and glia.

\section{Characterization of midline glia}

Having observed that the annulin-expressing midline glia are derived from the MNB, we then further characterized their morphology, using immunohistochemical staining for annulin protein to label glial cell bodies and processes. Figure $1 E$ shows the pattern of annulin-expressing glial cell bodies at $45 \%$ of development. The embryo in Figure $1 F$ was double stained for annulin and for an axonal marker recognized by the $8 \mathrm{~B} 7 \mathrm{MAb}$. It was photographed in a more ventral focal plane, revealing glial processes. We have defined six classes of midline glia based on their positions and morphology. These classes are as follows, from posterior to anterior (see Fig. $1 E, F$ ):

(1) The posterior midline glia (labeled p) have extensive posterior and lateral processes that extend to the medial edges of the longitudinal tracts and to the anterior midline glia of the next posterior segment. It is difficult to visualize the cell bodies of these glia because they are small and tightly apposed to the MNB. We estimate that there may be only one or two cells of this class per segment. 

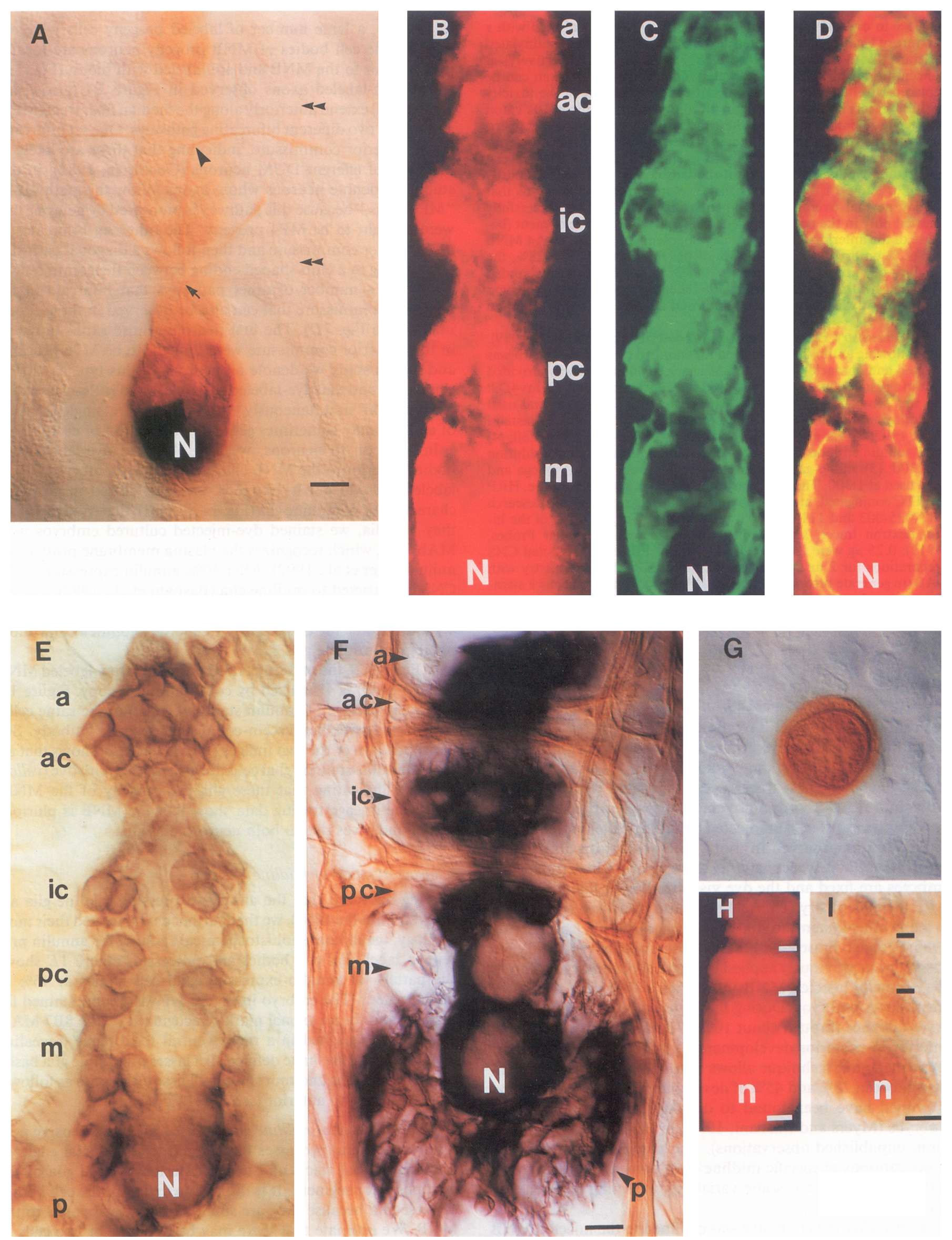
(2) The $M N B$ sheath glia (m) are lateral to the MNB cluster, and extend processes that enwrap the cell bodies of MNB progeny and the MNB itself. There are about six of these cells per segment.

(3) There are two posterior commisural midline glia (pc) on each side of the MFT, just posterior to the posterior commisure. They extend processes that enwrap posterior commissural axons.

(4) The intercommissural midline glia (ic) are located between the commissures. Their processes enwrap the commissures and ensheath the cell bodies of the MP3 progeny neurons. There are about six of these cells.

(5) The anterior commisural midline glia (ac) are similar to the posterior commissural midline glia. There are two of these cells on each side of the midline, just anterior to the anterior commissure, and their processes enwrap the axons of the anterior commissure.

(6) There are about 10 anterior midline glia (a). Their cell bodies are located just anterior to the class $5 \mathrm{glia}$, and they extend processes posteriorly to enwrap the commissures.

The cell bodies of the four classes of axon associated glia (pc, ic, ac, a) appear in their final locations at about the same time, suggesting that the different glial positions are populated simultaneously by migration. The overall pattern of orthopteran midline glia, as visualized with tracer dyes or by annulin staining, is very similar to that observed in Drosophila embryos (Fig. $1 H, I$; see Discussion).

\section{Neurons and glia are born in distinct temporal phases}

To characterize the temporal pattern of generation of neuronal and glial MNB progeny, we injected the MNB with tracer dyes at several developmental stages and cultured the injected embryos for varying lengths of time. When the MNB is injected at $28 \%$ and the embryos cultured until $32 \%$, round dye-filled progeny, which are likely to be GMCs or neurons, can be visualized just dorsal and anterior to it (Fig. 2A). If cultures of these embryos are maintained until $38 \%$, the round progeny are observed to extend axons that bifurcate in the anterior and posterior commisures (Fig. 2B). One pair of midline neurons in Figure 2 $A$ and $2 B$, are not labeled with lineage tracer. Examination of these unlabeled cells using Nomarski optics indicates that they are pioneer MP4-class neurons. The parent of these cells either is born from the MNB before $28 \%$ or is not derived from the MNB. The MP6-class neurons seen in Figure $2 B$ are the pioneers of this pathway, and are thus likely to be the same as the "MP6 progeny" described by Bate and Grunewald (1981). Similarly, the labeled MP4-class neurons in Figure $2 B$ are likely to correspond to the cells known as "MP5 progeny" (Bate and Grunewald, 1981). Thus, these midline pioneer neurons are actually derived from the MNB rather than from separate midline precursors. We have not been able to determine whether the "MP4 progeny," which pioneer the MP4-class pathway, are also generated from the MNB, because MNBs injected before $28 \%$ die or do not divide.

When the MNB is injected at $34 \%$ and the embryos cultured until $38 \%$, a very different pattern of progeny is observed. Instead of round GMC/neuronal progeny, cells with an elongated morphology, which are likely to be glia, are observed lateral to the MNB (Fig. 2C). Lightly stained glia can be also visualized in Figure $2 B$, in which the embryo was cultured from $28 \%$ to $38 \%$. In this panel, the glia are much fainter than the neurons, because progeny generated shortly after MNB injection incorporate more dye than do progeny generated later. When embryos injected at $34 \%$ are cultured until $44 \%$, the glia migrate anteriorly and take up positions around the commissures. In these embryos, MP4- and MP6-class axons are also observed, suggesting that a new set of neurons are generated after the glia are produced (Fig. $2 D$ ). It is unclear whether glial progeny continue to be generated after the second neuronal production phase begins.

\section{Neuronal development and engrailed expression at the CNS midline}

To characterize further the temporal and spatial patterns of development at the midline, we stained $27-45 \%$ embryo segments with MAb $8 \mathrm{~B} 7$, which labels neuronal cell bodies and axons. In these experiments, we simultaneously examined engrailed gene expression at the midline by double staining the embryos with $\mathrm{MAb}$ inv4D9, which recognizes the nuclearly localized engrailed protein (henceforth denoted simply engrailed; Patel et al., 1989). As described below, we find that in grasshopper embryos most or all of the MNB progeny express

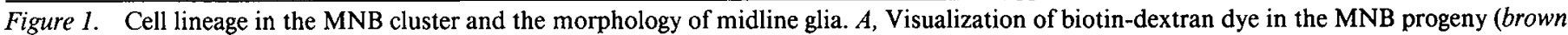

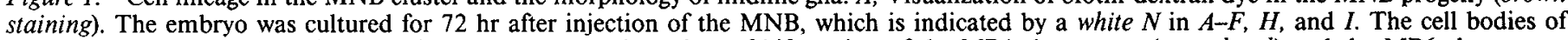

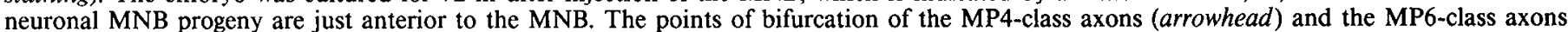

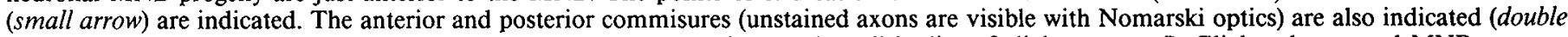

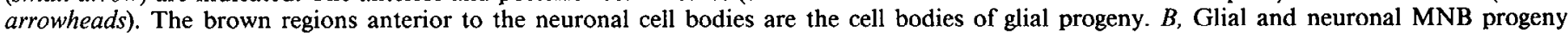

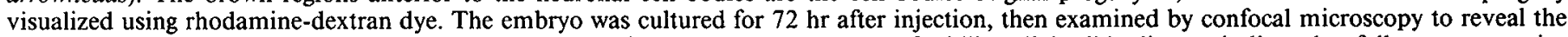

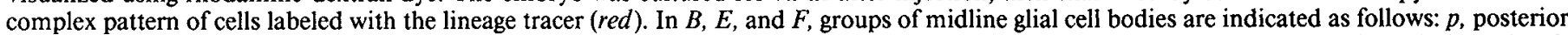

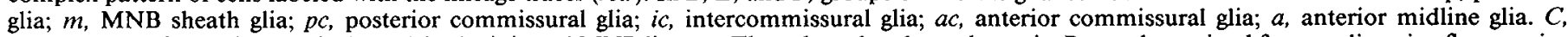

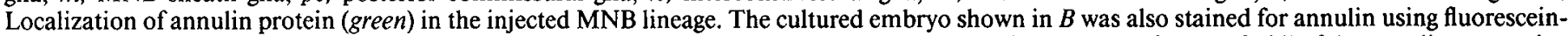

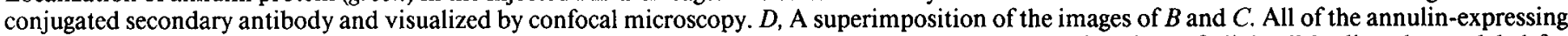

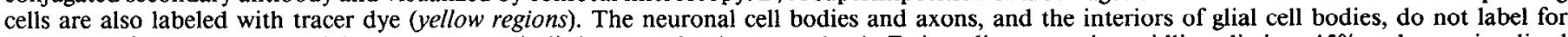

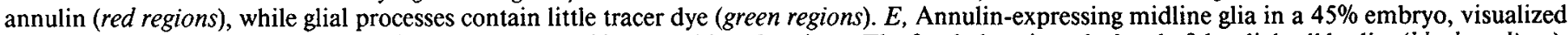

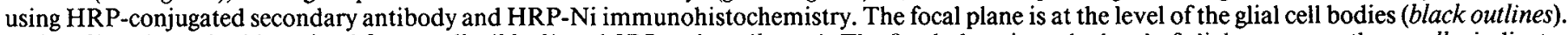

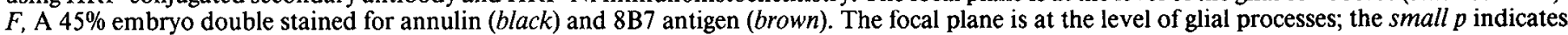

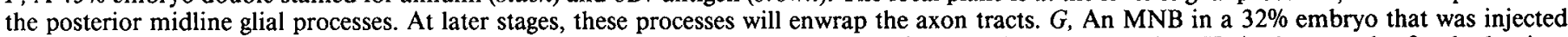

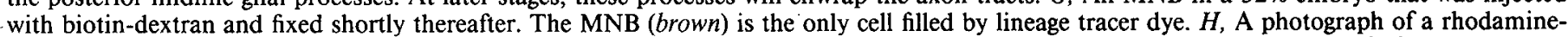

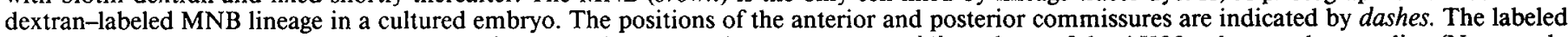

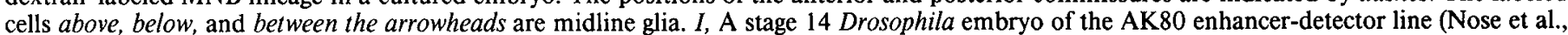

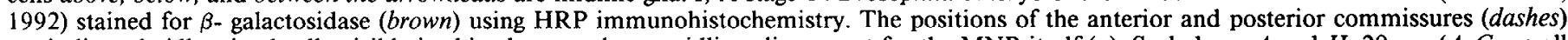

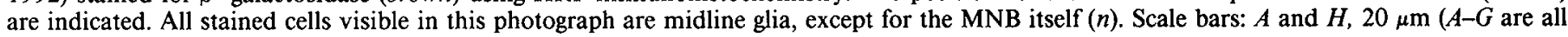
at the same magnification); $I, 5 \mu \mathrm{m}$. 

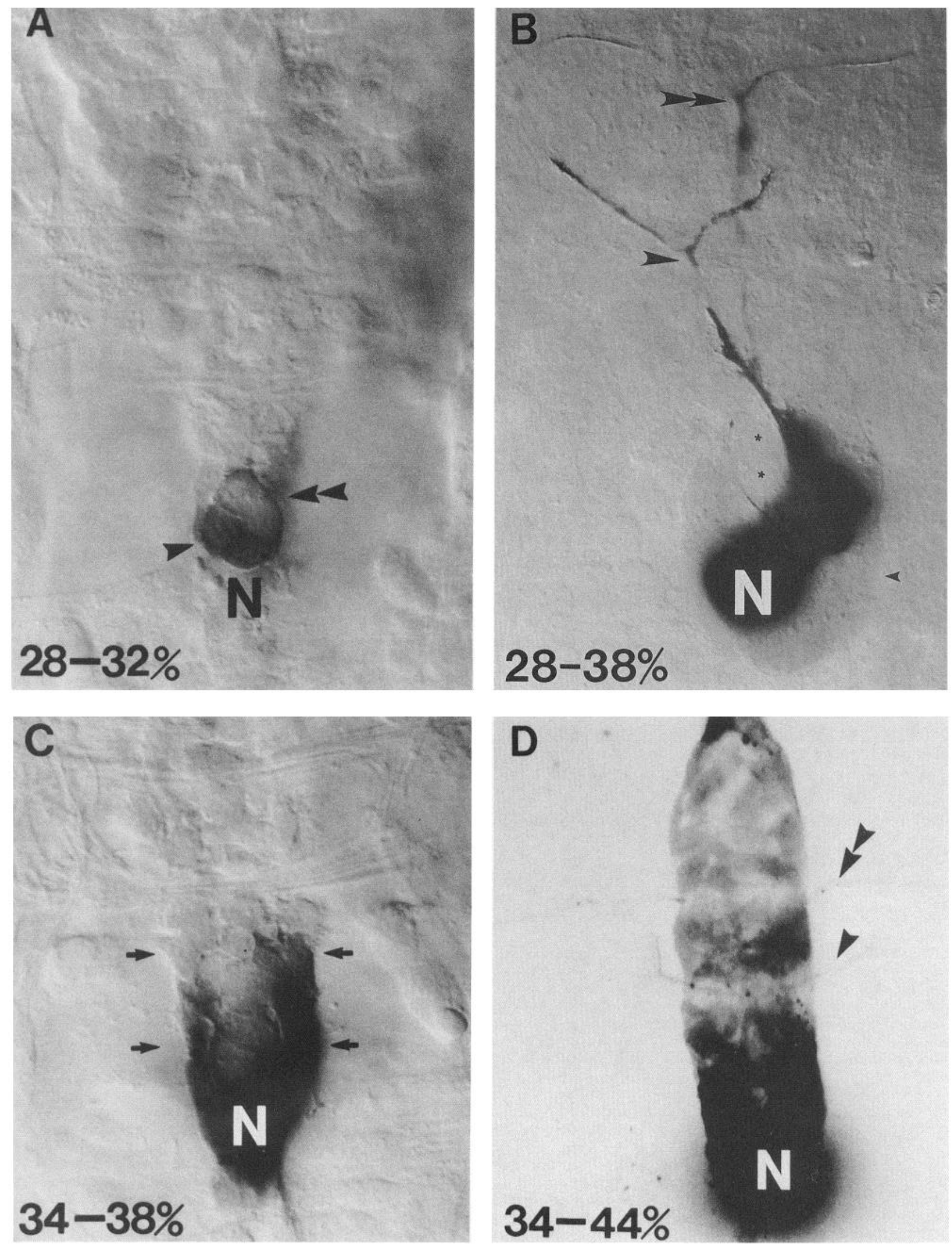

Figure 2. Midline neurons and glia are generated in distinct temporal phases. To identify the temporal order of appearance of MNB progeny, MNBs were injected with a mixture of rhodamine and biotin-dextrans at $28 \%$ or at $34 \%$, and the embryos cultured for different periods of time before fixation. In all cases, the MNB is marked with an $N$. In $A$, the MNB is below the plane of focus. $A-C$ are photographs, using Nomarski optics, of cultured embryos in which biotin tracer is visualized using streptavidin-HRP and HRP histochemistry. $D$ is a confocal microscope z-series 
engrailed shortly after they are born. Thus, double staining with inv4D9 and 8B7 provides an excellent method for visualizing the birth and differentiation of each cell in the MNB lineage (Fig. 3). The development of the MNB progeny, as determined by the lineage tracing and antibody staining experiments, is summarized in the schematic diagram of Figure 4.

At about $27 \%$ of development, three large engrailed-positive cells are observed at the midline, near the posterior segment boundary [Figs. $3 A$ (arrowheads), $4 A$ (en + cells are indicated by black nuclei in this figure)]. Engrailed staining then disappears from one of the large cells, and diminishes in intensity in the other two [Figs. $3 B$ (arrowheads), $4 B$ ]. At the same time, three smaller engrailed-positive cells appear, two of which are seen in Figure $3 B$ (arrows). We hypothesize that these smaller cells may be progeny of one or more of the large cells, as they are closely apposed to them. One of the three large cells (probably the one that turned off engrailed first) then enlarges further and moves dorsally to become the MNB (labeled "N"). The three small engrailed-positive cells are now located just anterior to the MNB [Figs. $3 C$ (arrows), $4 C$ ].

The other two large cells seen in Figure $3 A$ now switch off engrailed expression and remain ventral to the MNB cluster. We find that, after the MNB enlarges, these two cells can still be detected by staining with antibodies to the cell surface protein fasciclin I (Bastiani et al., 1987). They remain ventral to the MNB cluster until at least $40 \%$ of development (data not shown). We do not know their ultimate fates.

Each of the three engrailed-positive smaller cells seen in Figure $3 C$ now appear to divide once, and their progeny switch off engrailed expression and begin to express the $8 \mathrm{~B} 7$ antigen on their processes [Figs. $3 D$ (brown growth cones), $4 D$ (orange and red cells)]. They then extend axons along the midline that bifurcate in the anterior and posterior commissures. These axon trajectories are like those described earlier for neurons known as "MP4, 5, and 6 progeny" (Goodman, 1982). However, the lineage tracing experiments described above (Fig. $2 A, B$ ) show directly that the "MP5 progeny" and "MP6 progeny" neurons are actually derived from the MNB, and we suggest that this may be true for the "MP4 progeny" as well. Thus, our data are consistent with a model in which the three engrailed-positive smaller cells (Fig. 3C) are GMCs derived from the MNB rather than separate midline precursors. The cells previously identified as MP4, 5, and 6 may be the three large engrailed-positive cells seen in Figure $3 A$, or the three smaller cells of Figure $3 C$.

Shortly after the MP4 and MP6-class neurons extend axons, a number of other engrailed-positive cells appear adjacent to the MNB cluster [Figs. $3 D-F$ (arrowheads), $4 D, E$ (green cells with black nuclei)]. These new cells often have ellipsoidal nuclei and elongated shapes, while midline neurons are round and have circular nuclear outlines. The elongated cells are located in positions like those of the dye-filled progeny observed in Figure $2 C$, and their morphology suggests that they are midline glia or glia precursors. They express engrailed when they are adjacent to the MNB neuronal progeny, but then switch off expression after migrating anteriorly past them. They do not express the neuronal 8B7 antigen.

There is a long phase in which all cells appearing adjacent to the MNB have a glial morphology. No new 8B7-positive neurons appear along the midline between approximately $33 \%$ and $40 \%$ of development. However, after $40 \%$ new MNB cluster neurons appear whose axons follow a variety of pathways [Figs. $3 G, H$ (black cells and unstained cells); $4 G$ (blue and purple cells); see also Figs. $1 A, 2 D]$. The axons of efferent DUM neurons bifurcate in the anterior commissure and follow the tract pioneered by earlier MP4-class neurons (Figs. 1 $A, 2 D$; Goodman, 1982). MP6-class neurons, whose axons bifurcate in the posterior commissure, are also added after $40 \%$ (Figs. $1 A, 2 D$ ). We have made approximate counts of midline neuronal cell bodies in 8B7-stained embryos in order to determine the time course of neuronal production. At about $30 \%$ of development there are $3 \pm 1$ ( $N=18$ segments) round 8B7-positive cells anterior to the MNB; at $35 \%, 7 \pm 2(N=36)$; at $40 \%, 8 \pm 2(N=31)$; at $45 \%, 13 \pm 3(N=13)$; and at $50 \%, 21 \pm 2(N=9)$. (Note that since GMCs stain for engrailed, but are probably $8 \mathrm{~B} 7$-negative, there is a time lag between the appearance of engrailed-positive and 8B7-positive cells.) These data are consistent with the early birth (before 33\%) of three or four GMCs that each divide once to produce six to eight midline pioneer neurons. Glia or glia precursors are then generated until about $40 \%$, and no new neurons are added during this time. After $40 \%$, neuronal production begins again, and is likely to continue through most of the remainder of embryogenesis, since 80-90 progeny are generated by hatching (Thompson and Siegler, 1991). Given the 5 hr division cycle characteristic of grasshopper neuroblasts (Shepherd and Bate, 1990), $200 \mathrm{hr}$ or $40 \%$ of development would be required to produce $40 \mathrm{GMCs}$.

Some of the neuronal progeny of the MNB turn off engrailed expression shortly after they are born, but a subset of midline neurons continue to express engrailed in $45 \%$ and $50 \%$ embryos [Figs. $3 G, H$ (black cells); $4 G$ (orange and blue cells with black nuclei)]. The cell bodies of the engrailed-positive neurons are small and located ventrally in the cluster. Examination of the 8B7-stained axon fascicles extending anteriorly from their cell bodies suggests that these neurons are of the MP6 class. The

image of the fluorescent rhodamine tracer. The confocal image was used because it is impossible to obtain a photograph of a $44 \%$ embryo in which all axon tracts and glia are in focus, due to the three-dimensional nature of the MNB cluster at later stages. $A$, The MNB was injected at $28 \%$ of development, and the embryo cultured until 32\%. Two round GMC or neuronal progeny are visible on top of the MNB (single arrowhead). Note that two other neuronal cell bodies are not dye labeled, but can be visualized with Nomarski optics (double arrowheads); the black line at the edge of these cell bodies is dye in the injected MNB, which is below the plane of focus. $B$, The MNB was injected at $28 \%$ and the embryo cultured until $38 \%$. The axons and cell bodies of MP6-class (single arrowhead) and MP4-class (double arrowheads) neurons are visible. There are at least two unlabeled neuronal cell bodies between the left-hand arrow and the dye-filled progeny (asterisks). Lightly staining glia are also present around the MNB (region posterior to the arrows). $C$, The MNB was injected at $34 \%$ and the embryos cultured until $38 \%$. Only cells with a glial morphology are labeled under these conditions (arrows). Unstained neuronal cell bodies can be visualized in the midline region between the arrows and above the $N$, however. The unstained anterior and posterior commissures are also visible. $D$, The MNB was injected at $34 \%$ and the embryo cultured until 44\%. Dye-labeled MP4-class (double arrowheads) and MP6-class (single arrowhead) axons are visible. The MP4-class axons bifurcate in the anterior commissure, and the MP6-class axons in the posterior commissure. Note that these MP6-class axons follow a different pathway from the ones seen in Figure $1 A$. These axons are extending branches anteriorly in the longitudinal connectives. Glial labeling (gray staining) is visible along the entire midline, indicating that the glia seen in $C$ have migrated anteriorly to their final positions. The black spot at the top of $C$ is dye from the MNB in the next anterior segment, which was also injected in this experiment. 

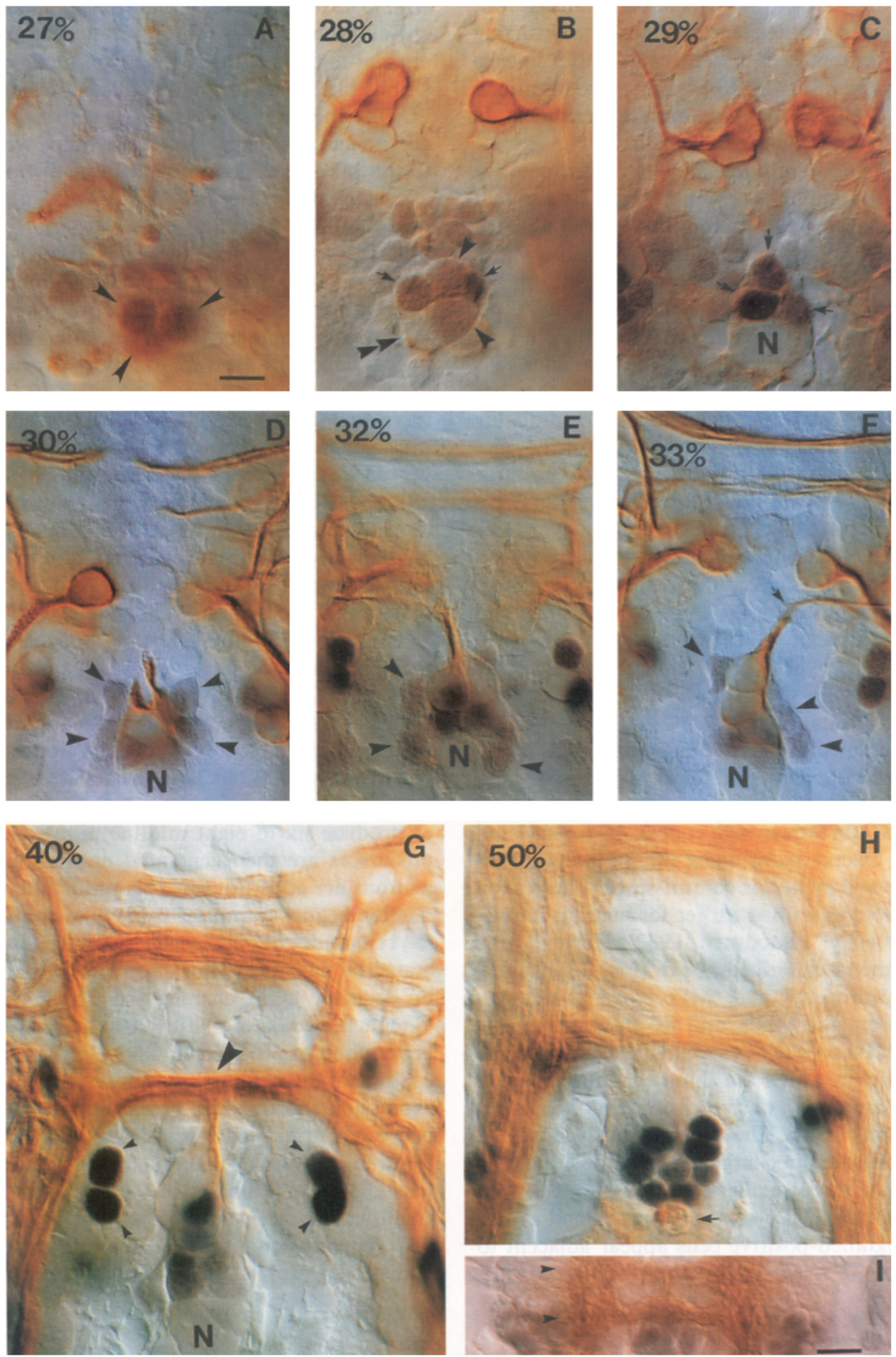

Figure 3. Neuronal development and engrailed expression at the CNS midline. In $A-H$, grasshopper embryos are stained for both the neuronspecific 8B7 antigen (brown) and for engrailed, using the inv4D9 MAb (black). A, The posterior midline of the grasshopper CNS at 27\% of development. The three large engrailed-expressing cells (arrowheads) are indicated. The lightly staining nuclei in $A-C$ are those of ectodermal cells in the posterior compartment, all of which express engrailed at this stage. $B$, At $28 \%$ of development, one of the three large cells seen at $27 \%$ has stopped expressing engrailed (double arrowhead) while the other two continue to do so (single arrowheads). Small engrailed-expressing cells (arrows) now appear over the large cells. $C$, By $29 \%$ of development, the two other large, initially engrailed-positive cells have moved ventral to the MNB and are not visible. Three small engrailed-expressing cells (small arrows) are located immediately anterior to the MNB (indicated by $N$ in $C-G$ ), which does not express engrailed. These small cells are probably GMCs that divide to produce the early MP4-class and MP6-class neurons. The MNB has enlarged to its final size. D. At 30\% of development at least two neurons in the MNB cluster have begun expressing $8 \mathrm{~B} 7$ antigen and are extending axons anteriorly (note brown growth cones). These are likely to be MP4-class neurons, which pioneer the MFT. Another class of engrailedexpressing cells now appear lateral to the MNB cluster (arrowheads). These cells have irregular shapes and elongated nuclei, and express engrailed at lower levels than the GMCs of $C$. Two of the pioneer pathways of the anterior commisure can be seen at the top of $D$. $E$, At $32 \%$ of development, engrailed-expressing cells (arrowheads) continue to appear anterior and lateral to the MNB. The pioneer growth cones of the MFT, extending anteriorly from the MNB cluster, have reached the point where the posterior commisure will form. The darkly staining engrailed-positive cells at 
overall pattern of engrailed-expressing neurons is quite similar between grasshopper and Drosophila embryos (compare Fig. $3 H, I$; Patel et al., 1989).

\section{Cell divisions at the CNS midline}

Although the lineage tracing experiments showed that the MNB gives rise to all of the classes of midline glia, we could not determine from these data whether the glia are immediate offspring of the MNB or of MNB-derived glioblasts. The lineage tracing (Fig. $2 A-C$ ) and antibody staining experiments (Fig. $3 D-$ $F$ ) also showed that $\mathrm{GMC} /$ neuronal $\mathrm{MNB}$ progeny emerge from the dorsoanterior flank of the MNB, while glial (or glial precursor) progeny emerge laterally. We wished to determine whether this was because the MNB's mitotic aster has different orientations during the neuronal and glial production phases. To answer these two questions, we examined the pattern of mitoses at the midline using antibodies to $\beta$-tubulin. Staining for $\beta$-tubulin allows visualization of the mitotic aster, and by examining a large number of stained embryos at various stages of development one can determine the location and timing of cell division events, as well as the orientation of the aster during each division. This method has been used to examine the pattern of mitoses during early embryogenesis in Drosophila (Foe, 1989).

In the grasshopper CNS, we are able to localize precisely cell divisions relative to the positions of the axon tracts, because axonal microtubules are also visualized by $\beta$-tubulin staining. Of 308 clearly stained MNBs, 41 were in mitosis in 33-45\% embryos. We find that only three types of mitoses are seen at the midline. First, we observe MNB divisions, and the frequency of these is consistent with the $5 \mathrm{hr}$ cycle time of grasshopper neuroblasts (Shepherd and Bate, 1990). Figure $5 A$ is an optical cross section through the dorsoventrally oriented mitotic aster of an MNB at metaphase (arrowhead). Second, mitoses are observed in small cells dorsal and slightly anterior to the MNB, and asters are oriented in the horizontal plane during these divisions [Figs. $5 D$ (arrowhead), $6 A$ ]. Based on their position (see Fig. $2 A$ ), these cells are likely to be GMCs, which divide once to produce two neuronal progeny. Third, we observe mitoses near the commissural tracts. Dividing cells are located either between the anterior and posterior commissures or immediately dorsal to commissural axons [Figs. $5 C$ (arrowheads), $6 A$ (GLIA)]. The dividing cells are likely to be glial precursors, because they are located in the regions where migrating MNB progeny are observed in lineage tracing experiments (Fig. 2C), and they are among the most dorsal cells in the CNS. The overall pattern of mitoses that we have observed is summarized in the diagram of Figure $6 \mathrm{~A}$.

In segments older than $33 \%$ of development, the MNB's mitotic aster is always oriented dorsoventrally during metaphase, as seen in Figure $5 \mathrm{~A}$. However, after cytokinesis, MNB progeny can emerge either dorsally (and slightly anterior), or laterally. This is because the aster can rotate during telophase to allow cytokinesis to occur in a lateral direction. Cytokinetic events of this type are shown in Figure 5, $B$ and $5 E$ (arrowheads). In Figure $5 B$, the progeny cell is lateral and slightly posterior to the MNB. We find that the MNB undergoes lateral cytokinesis between approximately $33 \%$ and $40 \%$ of development. After $40 \%$, cytokinesis occurs primarily along the dorsoventral axis. The two orientations of MNB mitoses are depicted in the diagram of Figure $6 B$. The timing of the developmental switch between the two types of cytokineses suggests that lateral cytokinesis gives rise to glia and/or glial precursors, while dorsal cytokinesis produces GMCs. This correlates well with the positions in which glial (elongated, 8B7-negative) and neuronal (round, 8B7-positive) cells initially appear (Figs. $2 A, C ; 3 D-F$ ).

Finally, $\beta$-tubulin staining shows that many of the lateral neuroblasts also divide both dorsoventrally and laterally. The mitutic aster can be oriented in the horizontal plane at metaphase during the lateral divisions, which are often synchronized across the midline (Fig. $5 F$, single arrowheads).

\section{Discussion}

Many cell lineages have been traced in vertebrate nervous systems using dye labeling or retroviral markers, and these experiments have provided evidence for precursors that give rise to both neurons and glia and remain undetermined until late in development (Turner and Cepko, 1987; Holt et al., 1988; Galileo et al., 1990; Leber et al., 1990; Bronner-Fraser and Fraser, 1991). Even small clones are often observed to contain both neurons and glia. It has also recently been reported that a $\mathrm{Dro}-$ sophila lateral neuroblast produces glial progeny (Udolph et al., 1993).

In this report, we show that the orthopteran MNB is a multipotent progenitor cell, and that it produces neuronal and glial progeny in distinct temporal phases. In its first phase, the MNB generates midline pioneer neurons. Later in development, it gives rise to a large number of midline glia. Some of these ensheath the MNB cluster, while others migrate anteriorly and enwrap the commissural axons. Finally, there is a second neuronal production phase, during which several classes of midline neurons are generated (summarized in Fig. 4). The transitions between these phases are correlated with changes in the direction of MNB cytokinesis (summarized in Fig. 6).

\section{Development of the midline neurons}

Neurons in the MNB cluster have axons that grow anteriorly along the MFT and bifurcate either in the anterior commissure (MP4-class neurons) or in the posterior commissure (MP6-class

the lateral edges of the panel are neurons that are not derived from the MNB; they are likely to be the "NH cells." $F$, By $33 \%$ of development, one of the Q1 pioneer growth cones (Myers and Bastiani, 1993b) has arrived at the midline (arrow), and a pioneer MFT axon has fasciculated with the Q1 axon. Three irregular engrailed-expressing cells are indicated by arrowheads. One of these appears to be migrating anteriorly past the MNB cluster. $G$, By $40 \%$, all MNB progeny lateral to the neuronal cell body cluster have switched off engrailed. New engrailed-positive GMCs, and possibly some neurons, have appeared in the cluster. The cells that express engrailed have small nuclei and are located ventrally in the cluster (nuclei are in the same focal plane as the MP6-class axons). The point of bifurcation of the MP6-class axon pathway is indicated (large arrowhead); the MP4-class pathway is in a more dorsal focal plane. The small arrowheads indicate the lateral engrailed-expressing neurons that are also visible in $E$ and $F . H$, At $50 \%$ of development, the engrailed-expressing cells are located in the ventral region of the cluster. Note the dead cell (arrow); cell death is often observed just posterior of the MNB at this stage. $I$, The Drosophila CNS engrailed pattern. Engrailed expressing midline cells (black) are stained with inv4D9 and CNS axons with MAb BP102 (brown). The commissures are indicated (arrowheads). Scale bars: $A, 20 \mu \mathrm{m}$ for $A-H ; I, 5 \mu \mathrm{m}$. 

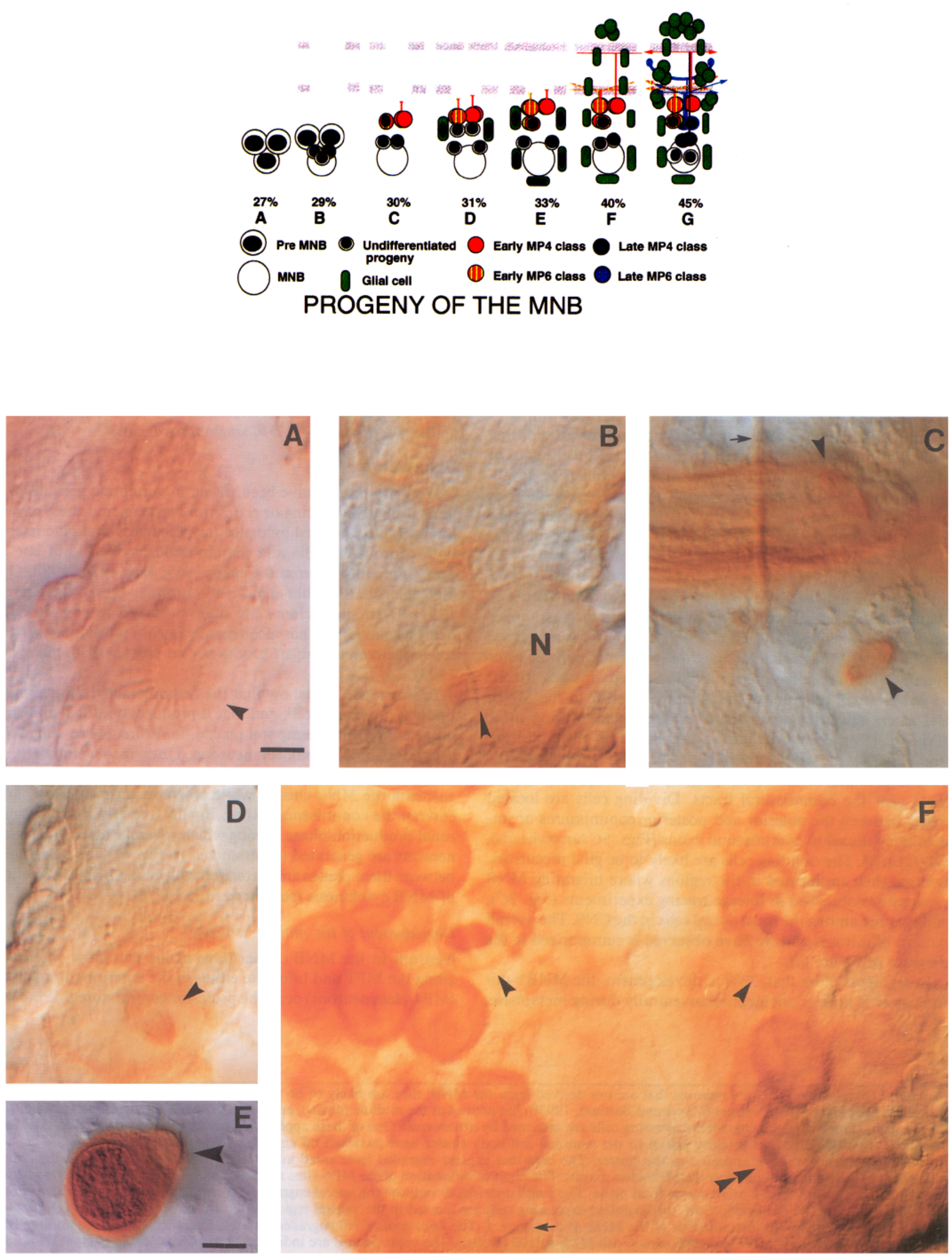
neurons). These bifurcating pathways are established by three pairs of pioneer neurons that were originally thought to be derived from midline precursors denoted MP4, MP5, and MP6 (Bate and Grunewald, 1981; Goodman, 1982). However, we have been able to show that at least two of these pairs of midline pioneers are actually derived from the MNB (Fig. $2 A, B$ ). The pioneer neurons are born before $33 \%$ of development, and no other neurons appear in the cluster until about $40 \%$.

After 40\%, neurons whose axons follow a number of different pathways are born. One group are MP4-class neurons whose axons bifurcate in the anterior commissure and exit the CNS (Figs. $1 A, 2 D$ ). Other neurons have axons that follow several different MP6-class pathways. One of these (Fig. 1 $A$ ) appears to terminate between the commissures, while another (Fig. $2 D$ and data not shown) extends along the connectives but remains within the CNS. Figure $2 D$ shows that new axons appear after $40 \%$, but does not directly prove that more neurons are added to the cluster after the glia are generated, since axonogenesis of some neurons could be delayed. However, the number of $8 \mathrm{~B} 7$ positive neuronal cell bodies within the cluster increases from 8 to 21 between $40 \%$ and 50\% (see Results), and 80-90 MNB progeny are generated by hatching (Thompson and Siegler, 1991). Thus, a second round of neurogenesis from the MNB must occur after the glial production phase.

Correlation of our data with previous analyses of CNS pathways suggests that the MP4-class cells that appear after $40 \%$ are likely to be efferent DUM neurons (Goodman, 1982). The MP6class neurons that we observe at $40-50 \%$ could correspond to some of the local and intersegmental interneurons identified in the adult by Thompson and Siegler (1991). We find that MP4and MP6-class axons appear during the same time interval (see Figs. $1 A, 2 D$ ). Based on the positions of their cell bodies within the MNB cluster, it was assumed in earlier studies that the first 12 progeny of the MNB are all efferent DUMs (Goodman, 1982). Ncuronal soma appcar to migrate within the cluster, however, so that birth order cannot be directly inferred from cell position.
The neurons that stably express engrailed are of the MP6 class, and expression persists in these cells until at least $50 \%$ of development (Fig. 3H).

\section{Development of midline glia}

Between the two phases of neuronal production, there is a long period during which the MNB generates only glia or glial precursors. Our data show that all of the annulin-expressing midline cell bodies are included within the collection of MNB progeny labeled by lineage tracer dyes (Fig. $1 B-D$ ). This indicates that all midline glia that can be visualized by annulin staining are derived from the MNB. It is possible that other classes of midline glia exist that do not express annulin, however. There are about 31 annulin-expressing midline glia per segment in a $45 \%$ embryo. Given the $5 \mathrm{hr}$ cell division cycle characteristic of grasshopper neuroblasts, all of the glia that we have identified could be produced within $10 \%$ of development if they are generated as precursors that divide once or twice (Shepherd and Bate, 1990). We observe mitotic events in the vicinity of the commissures that may represent divisions of migrating glial precursors (Fig. $5 C$; the pattern of mitoses at the CNS midline is summarized in Fig. 6A). In Drosophila, a precursor to the longitudinal glia has been described that divides a number of times (Jacobs et al., 1989). Glial precursors have also been characterized in Manduca (Copenhaver, 1993).

We have defined six types of midline glia based on annulin staining patterns. These fall into three major classes: axonal glia, sheath glia, and the posterior midline glia. The early axonal glia probably migrate anteriorly just behind the pioneer growth cones, and form a scaffold that may be essential for correct formation of the later commissural pathways.

The overall pattern of organization of the midline glia in Drosophila strongly resembles the pattern seen in the grasshopper embryo (Fig. $1 H, I$ ). However, there are fewer glia in fly embryos, at least at early stages of development. Only three pairs of axonal midline glia have been identified by electron

Figure 4. A schematic diagram depicting the development of the MNB lineage in the grasshopper embryo. Anterior is at the top and the temporal profile is from left to right. The approximate developmental stage in percent (for the T3 segment) is indicated below each section of the figure. The stippled boxes represent the anterior and posterior commissures. At $27 \%(A)$, three large engrailed-expressing cells (staining for engrailed protein is indicated by black nuclei) lie at the dorsal surface of the posterior mesectoderm of each segment. By $29 \%(B)$, one of the three large cells has switched off engrailed. The other two large cells turn down engrailed expression and move ventrally. Small cells appear over the cluster that strongly express engrailed. These are probably GMCs that give rise to MP4 and MP6-class neurons, which pioneer the MFT. At 30\% ( $C$ ), the MNB has enlarged to its final size. The early MP4-class and MP6-class neurons (red and orange) transiently express engrailed, but switch it off as they begin to express the neuronal $8 \mathrm{~B} 7$ antigen. At $31 \%(D)$, the anterior commisure forms. MP4-class and MP6-class neurons have extended growth cones. Engrailed-expressing glial progeny (green) also begin to appear. By $33 \%(E)$, the MP6-class growth cones have contacted the Q1 axons, and the posterior commissure forms. The MNB is producing only glia by this stage (the round clear cells with black nuclei in adjacent to the MNB in $D$ and $E$ are undifferentiated precursors, which will become glia). At $40 \%(F)$ several of the bifurcating pathways of the MFT have formed. The MP4class pathway $(\mathrm{red})$ bifurcates in the anterior commissure, while the MPG-class pathways (orange) bifurcate in the posterior commisure. The MNB probably ceases to produce glia at about this stage, and existing glia and glial precursors no longer express engrailed. Glial precursors are still dividing and migrating, however. By $45 \%(G)$, the glial groups have formed and glia have extended processes. The MNB is now generating only neuronal progeny. The progeny being produced at this time include both MP4-class (purple) and MP6-class (blue) neurons. The later MP4-class axons follow the bifurcating path in the anterior commissure established by the pioneer MP4-class axons (red). The neurons that express engrailed at this stage are of the MP6 class.

Figure 5. Mitoses at the CNS midline, as revealed by staining for $\beta$-tubulin. In $A-E$, embryos were stained with an antibody against $\beta$-tubulin followed by HRP immunohistochemistry $($ brown $)$ to allow visualization of microtubules. $A$, The MNB at metaphase, showing a dorsoventrally oriented mitotic aster (arrowhead), with the 22 kinetochores visible as a ring of dots. The focal plane is at the metaphase plate. $B$. A lateral MNB cytokinesis (arrowhead) in a 35\% segment. The progeny cell is emerging lateral and slightly posterior to the MNB (indicated by $N$ ). $C$, Two presumed glial precursors in metaphase in a $40 \%$ segment (arrowheads). The anterior mitotic aster is dorsal to the posterior commissural tract, while the posterior aster is just lateral to the cell bodies of neuronal MNB progeny. Both asters are oriented in the horizontal plane. The MFT is also indicated (arrow). D, A GMC (arrowhead) derived from the MNB (ventral to the focal plane) in metaphase in a $40 \%$ segment. The mitotic aster is oriented in the horizontal plane. $E$, Lateral division in an injected MNB. A $35 \% \mathrm{MNB}$ was injected with biotin-dextran and immediately fixed and stained. The neuroblast was dividing when injected, and the progeny cell (arrowhead) is emerging lateral to the MNB. $F$, Mitoses in the lateral NB array. NB 4.1 is in metaphase on both sides of the midline (arrowheads). NB 7.1 on the right side (double arrowheads) is also in metaphase, while its contralateral homolog is undergoing cytokinesis (arrow). Scale bars: $A, 10 \mu \mathrm{m}$ for $A-D ; E, 20 \mu \mathrm{m}$ for $E$ and $F$. 


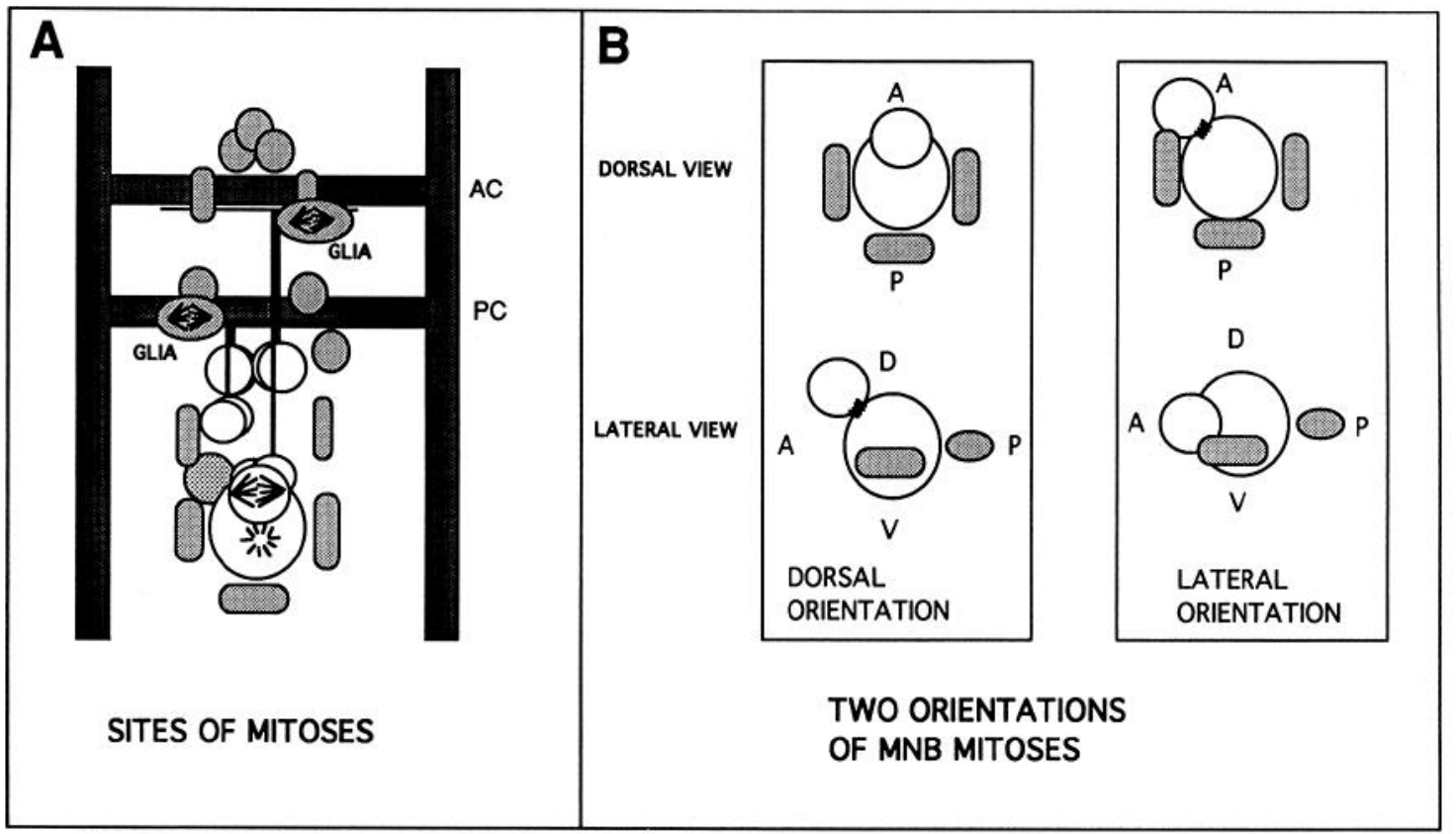

Figure 6. Schematic diagram of mitoses at the CNS midline. $A$, The sites and orientations of metaphase asters (indicated by schematic horizontal and dorsoventral asters) that are visualized at the midline between approximately $35 \%$ and $42 \%$ of development. Two glial precursor mitoses (GLIA), a GMC mitosis, and a dorsal MNB mitosis are depicted. The MNB and neurons are clear while glia are lightly stippled. The connectives and commisures $(A C, P C)$ are darkly stippled. B, The two types of MNB cytokineses. The dorsal orientation is seen after about $40 \%$ of development and is associated with production of neuronal progeny. The lateral orientation of cytokinesis is seen from about $33 \%$ until $40 \%$, and is associated with production of glia and glial precursors. The dorsal views of these cytokinetic events are the same as in $A$, while the lateral views are from the perspective of the longitudinal connectives. Glia are stippled, and the MNB and GMCs are clear. Anterior, posterior, dorsal, and ventral directions are indicated $(A, P, D, V)$.

microscopy (Jacobs and Goodman, 1989). The MGP (posterior) glia surround the posterior commissure, and the MGA (anterior) glia enwrap the anterior commissure. The MGM (middle) glia are located posterior to the anterior commissure, and their processes enwrap the anterior commissure and also cover the region between the commissures. In the grasshopper, there are approximately 24 axonal midline glia. About 10 of these are anterior midline glia, which are located anterior to the anterior commissure. These glia may be absent from Drosophila embryos, or they may appear later in development. There are four anterior commissural glia and four posterior commissural glia, which may be analogous to the Drosophila MGAs and MGPs. The six grasshopper intercommissural glia may be equivalent to the MGMs.

The midline glial lineage in grasshoppers is quite different from the lineage proposed for axonal midline glia in Drosophila (Klambt et al., 1991). These authors generated enhancer-detector lines that express lacZ in cells at the CNS midline, and used staining for $\beta$-galactosidase activity to identify the midline glia in collections of fixed embryos from these lines. In their model, there are eight distinct midline precursors in stage 12 embryos, and the three pairs of midline glia are produced by a single division of three of these precursors. At this point, the three glial pairs are thought to be arranged in the order MGP, MGM, MGA, from anterior to posterior. To generate the final order of the glia, the MGMs must migrate posteriorly over the MGAs into a position between the commissures, while the MGPs migrate anteriorly into the next segment. By contrast, in the grasshopper embryo all of the axonal glia are derived from the MNB and migrate anteriorly to their final positions within the same segment (Fig. 2C,D and data not shown). No glia migrate across segment boundaries.

It seems unlikely to us that such apparently homologous glia are generated by a completely different lineage in the two insects, especially since many other lineages and axon pathways have been found to be conserved between grasshopper and Drosophila. In all cases examined to date homologous neurons have been found to be generated by the same neuroblast lineages (Doe, 1992b). We suggest that the three "MPs" that give rise to the six midline glia in Drosophila (Klambt et al., 1991) could actually be early MNB progeny that migrate anteriorly and switch off engrailed expression before dividing to produce the pairs of midline glia. The lineage of such precursors would be obscured by cell migration and changes in gene expression, and would thus be difficult to trace using molecular markers in fixed embryo collections. In a similar manner, we have found that in grasshopper embryos some of the midline pioneer neurons are derived from the MNB rather than from an MP. The lineage of these neurons could not easily be determined using only molecular markers, however, because they switch off engrailed and switch on $8 \mathrm{~B} 7$ antigen before extending axons. Another possibility is that some of the axonal glia in Drosophila have arisen as a later evolutionary development, because they may be involved in the splitting of the initially fused commissural tract (Klambt et al., 1991), an event that does not occur in grasshopper embryos. In order to determine conclusively the origin of the midline glia, it will be necessary to trace glial lineages directly in Drosophila. 


\section{Control of cell fate in the MNB lineage}

Our studies show that there are at least three phases of the MNB lineage prior to $50 \%$ of development: an early phase during which neurons are produced, a middle phase of glial production, and a second neuronal production phase after $40 \%$. There may be some overlap between these phases, although there are distinct periods during which only glia or only neurons are generated. The switch between the glial and second neuronal phases of the MNB lineage is correlated with a change in the orientation of cytokinesis. Although the mitotic aster is always oriented dorsoventrally at metaphase, when glia (or glial precursors) are being produced the aster rotates during telophase and progeny emerge laterally from the MNB. When neurons are being produced, the aster retains a dorsoventral orientation and progeny emerge dorsally (summarized in Fig. 6B). In lateral grasshopper neuroblasts, experiments in which the aster is mechanically rotated have suggested that the orientation of cytokinesis can influence the identity of neuroblast progeny (Yamashiki and Kawamura, 1986).

For lateral neuroblasts, the identities of their progeny neurons are thought to be determined by birth order within the lineage (Doe, 1992a). It is possible that this is the case for the MNB lineage, but such a control mechanism would be very complicated, given the large number of progeny with different identities that are generated. Our observations on midline cell divisions suggest a model in which the direction of MNB cytokinesis defines the approximate position of the progeny after birth at each stage of development. The final identity of a daughter cell might then be determined by signals from the other cells in its immediate neighborhood.

One component of the choice of cell fate by an MNB daughter is the homeodomain transcription factor encoded by the engrailed gene. Engrailed is expressed by all MNB progeny shortly after their birth, but its expression is then switched off in all glia and in some neurons. In later embryos, engrailed is stably expressed only by a subset of neurons in the MNB cluster. These observations suggested that engrailed could be involved in the initial choice of cell fate by MNB progeny as well as in the determination of the identity of a specific class of midline neurons. To evaluate this possibility, we used antisense techniques to block the early phase of engrailed expression in the MNB lineage. These experiments show that engrailed is required for acquisition of the glial fate (Condron and Zinn, 1993).

\section{References}

Bastiani MJ, Harrelson AL, Snow PM, Goodman CS (1987) Expression of fasciclin I and fasciclin II glycoproteins on subsets of axon pathways during neuronal development in the grasshopper. Cell 48: 745-755.

Bastiani MJ, Couet HGD, Quinn JMA, Karlstrom RO, Kotrla K, Goodman CS, Ball EE (1992) Position-specific expression of the annulin protein during grasshopper embryogenesis. Dev Biol 154:129-142.

Bate CM, Grunewald EB (1981) Embryogenesis of an insect nervous system II: a second class of neuron precursor cells and the origin of the intersegmental connectives. J Embryol Exp Morphol 61:317-330.

Bentley D, Keshishian H, Shankland M, Toroian-Raymond A (1979) Quantitative staging of embryonic development of the grasshopper, Schistocerca nitens. Embryol Exp Morphol 54:47-74.

Bronner-Fraser M, Fraser SE (1988) Cell lineage analysis reveals multipotency of some avian neural crest cells. Nature 355:161-164.

Bronner-Fraser M, Fraser SE (1991) Cell lineage analysis of the avian neural crest. Development S2:17-22.
Condron BG, Zinn K (1993) Perturbation of the grasshopper median neuroblast lineage. Soc Neurosci Abstr 19:861.

Copenhaver PF (1993) Origins, migration and differentiation of glial cells in the insect enteric nervous system from a discrete set of glial precursors. Development 117:59-74.

Doe CQ (1992a) The generation of neuronal diversity in the Drosophila embryonic central nervous system. In: Determinants of neuronal diversity (Shankland M, Macagno ER, eds), pp 119-154. New York: Academic.

Doe CQ (1992b) Molecular markers for identified neuroblasts and ganglion mother cells in the Drosophila central nervous system. Development 116:855-863.

Foe VE (1989) Mitotic domains reveal early commitment of cells in Drosophila embryos. Development 107:1-22.

Galileo DS, Gray GE, Owens GC, Majors J, Sanes JR (1990) Neurons and glia arise from a common progenitor in chicken optic tectum: demonstration with two retroviruses and cell type-specific antibodies. Proc Natl Acad Sci USA 87:458-462.

Gimlich RL, Braun J (1985) Improved fluorescent compounds for tracing cell lineages. Dev Biol 109:509-514.

Goodman CS (1982) Embryonic development of identified neurons in the grasshopper. In: Neuronal development (Spitzer NC, ed), pp 171-212. New York: Plenum.

Holt C, Bertsch TW, Ellis HM, Harris WA (1988) Cellular determination in the Xenopus retina is independent of lineage and birth order. Neuron 1:15-26.

Jacobs JR, Goodman CS (1989) Embryonic development of axon pathways in the Drosophila CNS. I. A glial scaffold appears before the first growth cones. J Neurosci 9:2402-2411.

Jacohs JR, Hiromi Y, Patel NH, Goodman CS (1989) Lineage, migration, and morphogenesis of longitudinal glia in the Drosophila CNS as revealed by a molecular lineage marker. Neuron 2:1625-1631.

Klambt C, Jacobs JR, Goodman CS (1991) The midline of the Drosophila central nervous system: a model for the genetic analysis of cell fate, cell migration and growth cone guidance. Cell 64:801-815.

Leber SM, Breedlove SM, Sanes JR (1990) Lineage, arrangement, and death of clonally related motorneurons in the chick spinal cord. $J$ Neurosci 10:2451-2462.

Myers PZ, Bastiani MJ (1993a) Cell-cell interactions during the migrations of an identified commissural growth cone in the embryonic grasshopper. J Neurosci 13:115-126.

Myers PZ, Bastiani M (1993b) Growth cone dynamics during the migration of an identified commissural growth cone. J Neurosci 13: 127-143.

Nose A, Mahajan VB, Goodman CS (1992) Connectin: a homophilic cell adhesion molecule expressed on a subset of muscles and the motoneurons that innervate them in Drosophila. Cell 70:553-567.

Patel NH, Martin-Blanco E, Coleman KG, Poole SJ, Ellis MC, Kornberg TB, Goodman CS (1989) Expression of engrailed proteins in arthropods, annelids and chordates. Ccll 58:955-968.

Shepherd D, Bate CM (1990) Spatial and temporal patterns of neurogenesis in the embryo of the locust (Schistocerca gregaria). Development 108:83-96.

Singer MA, Hortsch M, Goodman CS, Bentley D (1992) Annulin, a protein expressed at limb segment boundaries in the grasshopper embryo, is homologous to protein cross-linking transglutaminases. Dev Biol 154:143-159.

Thompson KJ, Siegler MV (1991) Anatomy and physiology of spiking local and intersegmental interneurons in the median neuroblast lineage of the grasshopper. J Comp Neurol 305:659-675.

Turner DL, Cepko C.L (1987) A common progenitor for neurons and glia persists in rat retina late in development. Nature 328:131-136.

Udolph G, Prokop A, Bossing T, Technau G (1993) A common precursor for glia and neurons in the embryonic CNS of Drosophila gives rise to segment-specific lineage variants. Development 118:765-775.

Yamashiki N, Kawamura K-Y (1986) Microdissection studies on the polarity of unequal division in grasshopper neuroblasts: II. Cell division in binucleate neuroblasts. Dev Growth Differ 28:603-609. 